\title{
Erratum to: Custom Implants in TKA Provide No Substantial Benefit in Terms of Outcome Scores, Reoperation Risk, or Mean Alignment: A Systematic Review
}

\author{
Eran Beit Ner MD ${ }^{1,2}$ (D), Saad Dosani MBChB 3 , Leela C. Biant BSc (Hons), MBBS, AFRCSEd, FRCSEd \\ (Tr\&Orth), MSres, MFSTEd ${ }^{4}$, Gwenllian Fflur Tawy PhD ${ }^{4}$
}

Received: 11 August 2021 / Accepted: 12 August 2021 / Published online: 6 September 2021

Copyright (C) 2021 by the Association of Bone and Joint Surgeons

$\mathrm{n}$ the study, "Custom Implants in TKA Provide No Substantial Benefit in Terms of Outcome Scores, Reoperation Risk, or Mean Alignment: A Systematic Review," there are errors in the Results section of the Abstract and the Results section of the main text. We would also like to correct data from a table so that it matches the text.

In the Results section of the Abstract, we incorrectly noted the number of studies evaluating patient-reported outcome measures (PROMs). We would clarify the Results section of the Abstract by replacing the first two sentences with: "There was no apparent advantage to custom implants in terms of PROM scores. Of the four studies evaluating PROM scores, one study reported better KSSFunction scores at 2-year follow-up [17], but in that study,

Erratum to: Clin Orthop Relat Res DOI: 10.1097/CORR.

0000000000001651.

The online version of this article can be found under 10 .

\section{7/CORR.0000000000001651.}

\footnotetext{
${ }^{1}$ Department of Orthopedic Surgery, Yitzhak Shamir Medical Center, Zerifin, Israel, affiliated with the Sackler Faculty of Medicine, TelAviv University, Tel-Aviv, Israel

${ }^{2}$ Manchester Orthopaedic Centre, Manchester University National Health Service Foundation Trust, Manchester, UK

${ }^{3}$ Division of Medical Education, the University of Manchester, Manchester, UK

${ }^{4}$ Division of Cell Matrix Biology and Regenerative Medicine, the University of Manchester, Manchester, UK

E. Beit Ner $\bowtie$, Department of Orthopaedic Surgery Yitzhak Shamir Medical Center Tel-Aviv University Tel-Aviv, Israel, Email:

eranbnster@gmail.com
}

there were no differences in the Knee Injury and Osteoarthrosis Outcome Score (KOOS), no difference in the Knee Score, and no differences in satisfaction scores for function, pain, or expectations; there was a small difference favoring custom implants in terms of the global satisfaction score ( 0.3 points on a 5 -point scale), which is of uncertain clinical importance. Another study reported better KSS scores at 6 weeks, but then no difference after that [15], the third study reported no difference [21], and the fourth reported inferior KSS scores for custom implants [22]."

In the Results section of the main text, we misinterpreted the results of Reimann et al., which was reference 17 in our study. The sentence that details the results of Reimann et al., should be: "The custom implant had superior results to the standard implant (KSS 164.5 versus $146.3 ; \mathrm{p}<0.002)$ at 2-year follow-up. The KSS-Function score was also greater in the custom group than in the standard implant group at 2-year follow-up (82 versus 68 ; $\mathrm{p}<0.0002)$."

In the Results section of the main text under Radiographic Results, the new generation of implant tibial slope, as reported in Meheux et al., which was reference 15 in our study, should match the data in Table 4. It should be " $5^{\circ}$ at $5.53^{\circ} \pm 3.9^{\circ}(\mathrm{p}=0.42)$."

The authors apologize for these errors. 